\title{
First Indian practice guidelines in cardiac surgery ... Never too late!
}

\author{
Om Prakash Yadava ${ }^{1}$
}

Received: 4 January 2019 / Accepted: 9 January 2019 / Published online: 29 January 2019

(C) Indian Association of Cardiovascular-Thoracic Surgeons 2019

Tongue in cheek, I dare say, it is a shame that 70 years down the independence, we still do not have any clinical practice guidelines for cardiac surgery in India, despite we being on cutting edge of technique and technology in the field and with spectacular advances under our belt. Not bemoaning the past, for it is counter-productive, an effort is being made to buck the trend. It is our pleasure to present the first Clinical Practice Guidelines on 'Follow up and Management of Valvular Heart Disease patients with Prosthetic Valve'. These guidelines are not only desirable, but are in fact mandatory, for a country like India for myriad of reasons:

i. We are genetically and racially different from the Caucasian population and pharmacogenomics teaches us that the response of the body to any drug, more so the anticoagulants, can be totally different in various ethnic groups.

ii. Our food habits involve consumption of high amounts of green leafy and coloured vegetables, fruits, herbs and spices, besides our cooking practices of deep frying and ubiquitous use of oils. This creates a fertile ground for various kinds of drug-food interactions, which we know can have a big bearing on anticoagulant action.

iii. The intestinal transit time of Indian population is much lower than the Caucasians and may affect the pharmacokinetics of anticoagulant drugs.

iv. Fasting as a religious practice is rampant in India, with its attendant consequences of disturbance in drug schedules and even abstinence.

v. Majority of people suffering from rheumatic valvular heart disease come from low socio-economic strata, where finances and other behavioural factors influencing compliance too chip in and play a spoilsport.

Om Prakash Yadava

op_yadava@yahoo.com

1 National Heart Institute, New Delhi, India vi. Diarrhoea, dysentery and gastrointestinal problems are endemic in our population, especially in the underprivileged class, thus making absorption of ingested anticoagulants unreliable.

vii. Limited and inequitable distribution of infra-structure for post-operative monitoring and follow up.

The aim of developing and publishing these clinical guidelines thus is to improve both the patient and the provider experiences. For the former, besides distributive justice through informed public policy, standardisation of practice is likely to improve the quality of care and patient outcomes. As for the latter, in the contemporary era of consumerism, with disconnection of 'patient-doctor' relationship, they provide sanctity and a measure of protection to the medical professionals, for all their well-intentioned, but potentially damaging, actions. This assumes utmost importance, given the nature of the field of 'Medicine', which is highly subjective and a moving target and is thus unpredictable.

For such and multiple other reasons, it makes sense to have our own guidelines. However, unfortunately, the indigenous data available is so tenuous and scanty that total de novo guidelines are not feasible and hence, an adaptation of existing European and American Guidelines, with suitable modifications, has been attempted in the Indian guidelines. Even though the international data has been reviewed in details, every section has its Indian recommendations to guide the clinician. Special subgroups like pregnant females, elderly, cancer patients, chronic kidney disease, heart failure, coronary artery disease, atrial fibrillation, and use of anticoagulants in the presence of complications like thrombo-embolic events, bleeding, thrombosis, and endocarditis have been very explicitly discussed. For the 'busybee' clinician, an executive summary has been provided in the beginning as a precis of the exhaustive document.

A caveat is that India is a vast and heterogeneous country with ethnicity, culture, food, beliefs, and practices changing every $100 \mathrm{~km}$. The avowed objective of the beneficial effects of the guidelines is contingent on their successful 
implementation. Local adaptation of these guidelines would therefore have to be made, based on local acceptability and ground realities, but that should never involve changing evidence-based core recommendations. Further, these are just 'guidelines' and not 'mandates' and blanket recommendations, ignoring patients' preferences can be disastrous. Shared responsibility should therefore continue to reign supreme, with the guidelines informing the decision-making, rather than being over-arching.
I sincerely wish this effort sets the ball rolling and we develop clinical practice guidelines for all facets of the practice of cardiac surgery. I also hope the readers and our fellow cardiac surgeons, cardiologists, and the physicians dealing with valvular heart disease find this document useful and guiding and that it informs their practice in a salutary way for the good of our 'patrons' and 'masters' —our 'patients'.

Publisher's note Springer Nature remains neutral with regard to jurisdictional claims in published maps and institutional affiliations. 